\title{
Fuel Economy and Naturalistic Driving for Passenger Road Vehicles
}

\author{
Xingda Yan \\ Energy Technology Research Group, \\ University of Southampton, \\ Southampton,UK \\ Email: xy1u16@soton.ac.uk
}

\author{
James Fleming \\ Energy Technology Research Group, \\ University of Southampton, \\ Southampton,UK
}

\author{
Roberto Lot \\ Energy Technology Research Group, \\ University of Southampton, \\ Southampton,UK
}

\begin{abstract}
The state-of-the-art eco-driving techniques does not take into account the naturalistic behaviour of human drivers. Therefore, in this paper, a unified driver model is proposed which describes the driver preference during car following and cornering cases. The model is formulated based on the optimal control theory. The fuel consumption model of a traditional vehicle with an internal combustion (IC) engine and CVT transmission is combined with the driver model. The proposed optimal controller is designed to generate speed profile and powertrain inputs, which give a compromise between the driver preference and fuel economy. The simulation results demonstrate that eco-friendly speed profile and optimal powertrain input trajectories could be selected which has good fuel economy and matches the driver desires.
\end{abstract}

\section{INTRODUCTION}

The energy exhaustion and environment pose great challenge on automobile industry. It become more urgent to effectively improve energy efficiency and reduce energy consumption of the motor vehicles. In addition to vehicular drive trains and mechanical systems impacting on fuel usage, the way a vehicle is driven can significantly influence overall fuel use and subsequent emissions. Research has estimated that $5-10 \%$ of fuel can be saved if drivers pursued a more fuel efficient, economical and environmentally friendly driving style referred to as eco-driving [1][2][3][4].

Schwarzkopf and Leipnik [5] adopted Pontryagin maximum principle to formulate optimal speed trajectories with minimized fuel consumption. An on-board controller is proposed to generate an optimal speed profile by Hellstrm et al. [6]. The road test has demonstrated a $3.5 \%$ reduction of fuel use over an entire route of $120 \mathrm{~km}$ without an increase in trip time. Model predictive control has been used by Kamal et al. [7] in order to develop a eco-driving, which can avoid inappropriate control inputs, such as unnecessary brakes or high control inputs, for realizing a comprehensive eco-driving assistance system. However, all these works fail to model the human drivers' preference for the speed profile optimisation. The efficacy of the eco-driving facilities is limited by the user acceptance. Even the most fuel economy speed profile is useless if the drivers feel uncomfortable to follow it. Burnham [8] has proposed a driver model to describe the naturalistic driver behaviour for car following case based on optimal control theory. Reymond etc. [9] proposed a cornering driver model, which gives the lateral acceleration limitation during curve driving. However, a unified driver model is need which could taken into the two most common driving scenarios: car following and cornering at the same time.

Therefore, the motivation of this paper is investigating the possibility of developing fuel optimization technique which meet the driver demands, such as travel time reduction, safety maximization, and ride comfort, in the mean time. The driver model proposed in this paper blended Burnham's and Reymond's model together. The combined optimization problem is solved by an orthogonal collocation method.

\section{DRIVER MODEL}

In this paper, the driver model is proposed based on a optimal control formulation [10] as follow.

$$
\begin{array}{rl}
\min _{u(t) \in \mathcal{U}} & J[\mathbf{x}, \mathbf{u}] \\
\text { s.t. } & \dot{x}=f(\mathbf{x}, \mathbf{u}, t) \\
& g(\mathbf{x}, \mathbf{u}, t) \leq 0 \\
& \text { Boundary Conditions }
\end{array}
$$

where the vector $\mathbf{x} \in \mathbb{R}^{n}$ represents the state of some physical system, which evolves according to the differential equation $\dot{\mathbf{x}}=f(\mathbf{x}, \mathbf{u}, t)$, and the vector $\mathbf{u} \in \mathbb{R}^{m}$ represents a control input. Therefore, in this formulation, the driver is modelled as a optimal controller which minimises the cost function $J[\mathbf{x}, \mathbf{u}]$, subject to the system dynamics (1), inequality constraints (2) and some boundary conditions (3) for the system state and input. This formulation has been chosen because is immediately compatible with fuel consumption minimisation models.

Burnham has proposed a optimal control based car following driver model in [8] which consist of differential equations and constraints to describe the driver's natural behaviour and a cost function to indicate the driver preference when following a lead vehicle. For human drivers, they always attempt to adjust their speed and headway distance by making a trade off between the urge to reduce trip time and maximize safety.

The driver model proposed here is based on a similar framework as Burnham's Model. Define the system state as $\mathbf{x}=\left[x_{e}, v_{e}\right]^{T}$ and the input as $\mathbf{u}=\left[a_{x}\right]$, where $x_{e}$ and $v_{e}$ are the travel distance and speed of the ego-vehicle respectively, $a_{x}$ the longitudinal acceleration. Therefore, the driver-vehicle 
system dynamics (1) can be described by following differential equations:

$$
\begin{aligned}
& \dot{x}_{e}=v_{e} \\
& \dot{v}_{e}=a_{x}
\end{aligned}
$$

If we assume that the travelled distance profile of the lead vehicle is given by a time series function $x_{l}(t)$, where the time $t$ is within a fixed period $[0, T]$, the headway distance between the ego-vehicle and the lead vehicle, which is a dependent variable, is calculated as:

$$
s=x_{l}(t)-x_{e}
$$

The driver cost can then be defined as:

$$
\begin{aligned}
J[\mathbf{x}, \mathbf{u}] & =\frac{1}{T} \int_{0}^{T} L_{D} \mathrm{dt} \\
L_{D} & =q_{v}\left(\frac{v_{e}}{v_{d}}-1\right)^{2}+q_{s}\left(\frac{\hat{s}}{s_{d}}\right)^{2}+q_{a}\left(\frac{a_{x}}{a_{\max }}\right)^{2}
\end{aligned}
$$

where $q_{v}, q_{s}$ and $q_{a}$ are three weighting factors, $v_{d}$ is the desired speed of the ego vehicle, which should be equal to the top speed of a specific car, and $a_{\max }$ is the maximum longitudinal acceleration.

For Burnham's model, $\hat{s}$ is equal to $\left(s-s_{d}\right)$, where $s_{d}$ is the desired headway distance for the reason of safety. However, this term will become relatively large or infinity when the headway distance $s$ is much larger than $s_{d}$. Therefore, the driver model will have unrealistic behaviour when the lead vehicle is far away or there is no traffic, as minimizing the term $\hat{s}^{2}$ will result in the ego-vehicle travelling as fast as possible to decrease $s$. Therefore, it leads to more natural behaviour if $\hat{s}$ is equal to zero when the headway distance $s$ is larger than $s_{d}$. In order to model this, an additional input variable $\hat{s}$ is introduced with the following constraint.

$$
\hat{s} \leq s-s_{d}
$$

$s_{d}$ can be calculated as $s_{d}=s_{d, 0}+T_{0} v_{e}$, where $s_{d, 0}$ is the minimum headway distance which the ego-vehicle should keep when it is stationary. $T_{0}$ is the driver's reaction time. Hence, the term $q_{s} \hat{s}^{2}$ will equal to $q_{s}\left(s-s_{d}\right)^{2}$ when the headway distance is smaller than the desired distance $s_{d}$ to avoid the collision into the lead vehicle, which is same as Burnham's paper [8]. But the term $q_{s} \hat{s}^{2}$ will be equal to zero when the headway distance is larger than the desired distance $s_{d}$, which means the driver behaviour will not be affected when the lead vehicle is relatively far away.

In order to model the driver behaviour when the ego vehicle is driving through a curved road section, a cornering driver model developed by Reymond et al. [9] is adopted, which adds an inequalities constraint on the lateral acceleration $a_{y}$ as follows

$$
a_{y}=\kappa\left(x_{e}\right) v_{e}^{2} \leq \Gamma_{\max }-\delta v_{e}^{2}
$$

where $\kappa\left(s_{e}\right)$ is the curvature, which is function of the traveled distance, $\Gamma_{\max }$ is maximum lateral acceleration, and $\delta$ is the driver's "safety margin" of error in their estimate of the curvature when approaching the corner. Based on the naturalistic driving data collected using a non-intrusive, portable, Automobile Data Acquisition Module developed by an EPRSC project at University of Southampton [11], sensible values of $\Gamma_{\max }$ are about $5-6 \mathrm{~m} / \mathrm{s}^{2}$ while $\delta$ should be around $4-5 \mathrm{rad} / \mathrm{km}$.

In summary, the optimal control formulation of the driver model is shown in the Table I where $v_{\max }$ is the speed limit; $s_{\text {min }}$ is the minimum headway distance; $a_{\text {min }}$ is the maximum deceleration; $x_{e, \text { init }}$ and $x_{e, \text { final }}$ are the initial and final travelled distance of the ego-vehicle; $L$ is the fixed total travelled distance.

TABLE I

Optimal Control Formulation of the Proposed Driver Model

\begin{tabular}{l|l}
\hline $\mathbf{x}$ & {$\left[x_{e}, v_{e}\right]^{T}$} \\
\hline $\mathbf{u}$ & {$\left[a_{x}, \hat{s}\right]^{T}$} \\
\hline$J[\mathbf{x}, \mathbf{u}]$ & $\frac{1}{T} \int_{0}^{T} L_{D} \mathrm{dt}$ \\
\hline$\dot{\mathbf{x}}=f(\mathbf{x}, \mathbf{u}, t)$ & $\dot{x}_{e}=v_{e}$ \\
& $\dot{v}_{e}=a_{x}$ \\
\hline \multirow{2}{*}{$g(\mathbf{x}, \mathbf{u}, t) \leq 0$} & $\hat{s} \leq s-s_{d}$ \\
& $a_{y}=\kappa\left(x_{e}\right) v_{e}^{2} \leq \Gamma_{\max }-\delta v_{e}^{2}$ \\
\hline & $0 \leq v_{e} \leq v_{\max }$ \\
& $0 \leq s_{\min } \leq x_{l}-x_{e}$ \\
Boundary Conditions & $a_{\min } \leq a_{x} \leq a_{\max }$ \\
& $v_{e}(0)=v_{e}(T)=v_{0}$ \\
& $x_{e}(0)=0$ \\
& $x_{e}(T)=L$ \\
\hline
\end{tabular}

Although the proposed driver model includes the two driving scenarios of car following and cornering in a unified way, these two driving cases are studied separately in order to clearly demonstrate the merit of the model.

\section{A. Case 1: Car Following}

In this case, it is assumed that the ego-vehicle is following a lead vehicle, which has a given speed profile and that both the lead and ego vehicle stay in a single lane, straight road $\left(\kappa\left(x_{e}\right)=0\right)$ as shown in Fig. 1, without any overtaking behaviour.

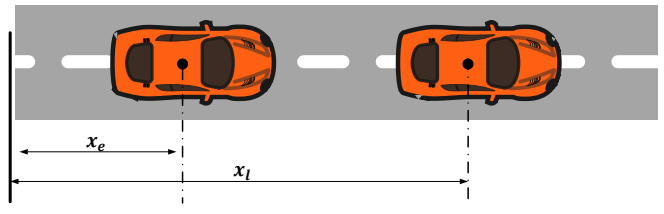

Fig. 1. Car Following Case

Hence, for the stage cost $L_{D}$ (8), minimising the term $\left(\frac{v_{e}}{v_{d}}-1\right)^{2}$ represents the urge to reduce the travel time. Minimising the term $\hat{s}^{2}$ means the driver would like to maintain a safe distance from the lead vehicle. The stage cost also considers minimising the vehicle acceleration for ride comfort by the term $q_{a} a_{x}^{2}$. Tuning the weighting factors $q_{v}, q_{s}$ and $q_{a}$ will make a compromise among travel time minimisation, safety maximisation and ride comfort. 


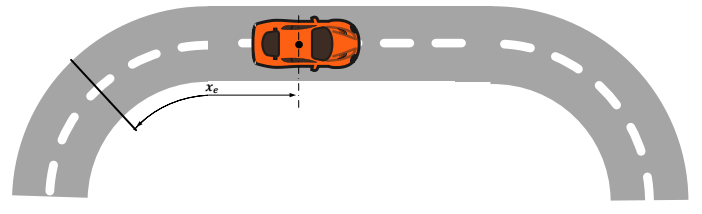

Fig. 2. Cornering Case

\section{B. Case 2: Cornering with No Traffic}

In this case, the road curvature $\kappa$ is given as the function of the ego-vehicle's travel distance $x_{e}$. And it is assumed that the lead vehicle is far enough away from the ego vehicle so that it does not affect the ego vehicle motion, and travels with same speed of the ego-vehicle.

$$
\begin{aligned}
v_{l} & =v_{e} \\
s & =s_{\max }
\end{aligned}
$$

We assumed that $s_{\max }$ is larger than $s_{d}$. Hence, the $\hat{s}$ is equal to zero, which means that the lead vehicle will have no impact on the behaviour of the ego vehicle. In other words, the ego vehicle travels with no traffic around.

\section{FUEL CONSUMPTION MODEL}

In this section, the dynamic model for the vehicle longitudinal motion and the fuel consumption of a traditional IC engine passenger car will be given.

\section{A. Driving Losses}

The overall traction force $F$ on the wheels can be calculated as

$$
\begin{aligned}
F & =m a_{x}+F_{a}+F_{r} \\
F_{a} & =\frac{1}{2} \rho A_{f} C_{d} v_{e}^{2} \\
F_{r} & =C_{r r} m g
\end{aligned}
$$

where $m$ is the vehicle mass, $F$ the force input to the powertrain, $F_{a}$ is the aerodynamic resistance force, $F_{r}$ the rolling friction force, $\rho$ the air density, $A_{f}$ the frontal area, $C_{d}$ the coefficient of drag and $C_{r r}$ the coefficient of rolling resistance.

\section{B. Internal Combustion Engine powertrain}

For the powertrain, a traditional passenger car is considered with a 2.0 litre internal combustion engine and a CVT transmission. The internal combustion engine model is implemented as a lookup table that maps engine torque $T_{e}$ and crankshaft speed $\omega_{e}$ to the fuel mass flow rate $q_{f}$ as shown in Fig. 3. Hence we may consider the fuel mass flow rate $q_{f}$ as a single mathematical function

$$
q_{f}=h\left(\omega_{e}, T_{e}\right)
$$

In order to correlate the engine speed with the vehicle speed, the CVT ratio $N_{g}$ is considered as an additional system input

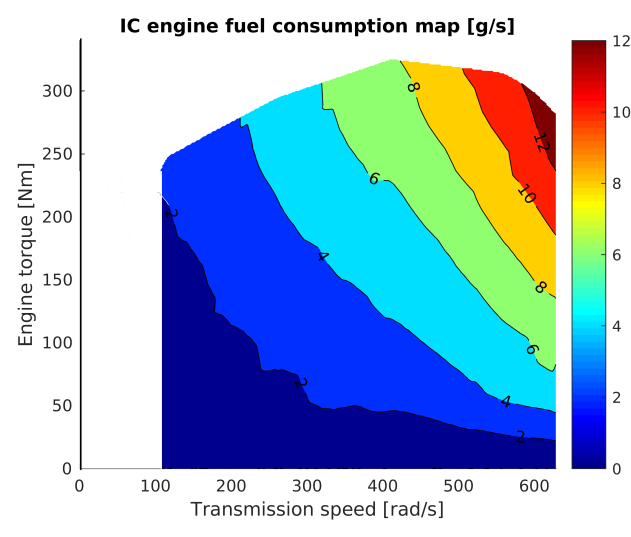

Fig. 3. Fuel mass rate map for the IC engine

with a boundary condition $N_{g} \in[4,10]$. Hence, the engine torque and crankshaft speed can be calculated as:

$$
\begin{aligned}
\omega_{e} & =\max \left(\frac{v_{e}}{r_{w}} N_{g}, 100\right) \\
T_{e} & =\frac{\max (F, 0)}{N_{g}} r_{w}
\end{aligned}
$$

where $r_{w}$ is the radius of the wheels and the engine has a idle speed of $100 \mathrm{rad} / \mathrm{s}$.

Substituting (13)(15)(16) into (14), the fuel mass rate becomes a function of the system state and input.

$$
q_{f}=\hat{h}\left(v_{e}, a_{x}, N_{g}\right)
$$

In addition, the maximum engine torque is given as a polynomial function of the engine speed $T_{\max }\left(\omega_{e}\right)$. This limitation is incorporated by the following inequality constraint in the optimal control formulation:

$$
T_{e} \leq T_{\max }\left(\omega_{e}\right)
$$

\section{Fuel ECONOMy ANd Driver Preference}

For the majority of human drivers, the objective of driving will be travelling to the destination safely and comfortably within a reasonably short time and paying less for the fuel. Therefore, in this section, a combined optimization problem will be established which will trade off the driver preference and fuel economy.

For the combined optimization problem, the system state of the driver-vehicle system is $\mathbf{x}=\left[x_{e}, v_{e}\right]^{T}$, while the control input is $\mathbf{u}=\left[a_{x}, \hat{s}, N_{g}\right]^{T}$.

The combined cost function $J[\mathbf{x}, \mathbf{u}]$ for this problem is defined as follows

$$
J[\mathbf{x}, \mathbf{u}]=\frac{1}{T} \int_{0}^{T}\left(w_{1} L_{D}+w_{2} q_{f}\right) d t
$$

where $w_{1}$ and $w_{2}$ are the two weighting factors for the driver cost and for the fuel economy minimization respectively. Hence, for a fixed $w_{2}$, larger $w_{1}$ will result in a solution with a smaller mean $L_{D}$ and a larger mean $q_{f}$ and vice versa. 
Compared to the driver model formulation, the combined optimization problem also include the additional inequality constraint (18) from the fuel consumption model.

In this paper, the Legendre-Gauss-Radau (LGR) pseudospectral method is adopted to solve the optimization problem numerically, which is featured by the general purpose optimal control software (GPOPS-II) [12]. The orthogonal collocation method is used by this Matlab software to generate the LGR points. The main advantage of GPOPS-II is that an adaptive mesh refinement algorithm is employed to iteratively adjust the number of mesh intervals, the width of each interval, and the polynomial degree. In the following subsections, the optimization problem is solved under different boundary conditions to investigate the benefit of the proposed problem formulation. Firstly, the model parameters and optimization boundaries which are identical in different scenarios are presented in Table II.

TABLE II

LIST OF PARAMETERS

\begin{tabular}{l|l|l}
\hline $\begin{array}{l}\text { Powertrain } \\
\text { Parameters }\end{array}$ & $\begin{array}{l}\text { DriverModel } \\
\text { Parameters }\end{array}$ & $\begin{array}{l}\text { Optimization } \\
\text { Boundaries }\end{array}$ \\
\hline \hline$m=1200 \mathrm{~kg}$ & $q_{v}=1$ & $s_{\max }=3000 \mathrm{~m}$ \\
\hline$\rho=1.225 \mathrm{~kg} / \mathrm{m}^{3}$ & $q_{s}=1$ & $s_{\min }=5 \mathrm{~m}$ \\
\hline$A_{f}=2 \mathrm{~m}^{2}$ & $q_{a}=1 e-2$ & $a_{\min }=-4 \mathrm{~m} / \mathrm{s}^{2}$ \\
\hline$C_{d}=0.35$ & $T_{0}=2 \mathrm{~s}$ & $a_{\max }=2.5 \mathrm{~m} / \mathrm{s}^{2}$ \\
\hline$C_{r} r=0.01$ & $s_{d, 0}=10 \mathrm{~m}$ & $v_{\max }=50 \mathrm{MPH}$ \\
\hline$\rho_{\text {petrol }}=0.75 \mathrm{~kg} / \mathrm{L}$ & $\Gamma_{\max }=5 \mathrm{~m} / \mathrm{s}^{2}$ & \\
\hline $4 \leq N_{g} \leq 10$ & $\delta=5 \mathrm{rad} / \mathrm{km}$ & \\
\hline
\end{tabular}

In order to reflect how each solution is close to the naturalistic driving, we defined the deviation of the naturalistic driving index as:

$$
\Pi_{\text {nature }}=\frac{\int_{0}^{T} L_{D} d t}{x_{e}(T)}[1 / m]
$$

It should be noted that a smaller value of this index means that the driver will feel more comfortable during this driving trajectory. And for the total fuel consumption is converted to the average fuel consumption in the common used unit $L / 100 \mathrm{~km}$.

$$
\Pi_{\text {fuel }}=\frac{\int_{0}^{T} q_{f} d t}{x_{e}(T) \rho_{\text {petrol }} 100}[L / 100 k m]
$$

In the following part, it demonstrated that how fuel minimization strategy affects the naturalistic driving behaviour of the human in different driving situation.

\section{A. Case 1: Car-following}

In this part, the speed trajectory of the lead vehicle is given. For simplicity, we assume the speed profile of the lead vehicle is featured as a five segment structure: stationary, acceleration, constant top speed, deceleration and stationary. It is assumed that the top speed of the lead vehicle is $30 \mathrm{MPH}$ as shown in Fig. 5 with the magenta dashed line. The total travel time is $60 \mathrm{~s}$, the total travel distance is $400 \mathrm{~m}$, and the initial and final headway distance are both $10 \mathrm{~m}$. While the weighting factor

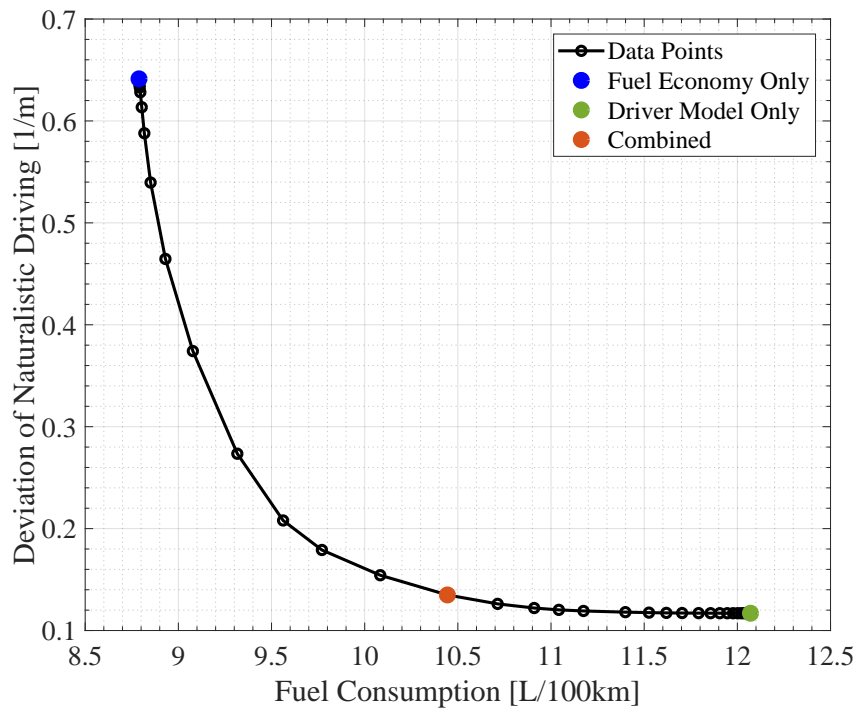

Fig. 4. Trade-off of two weighting factors
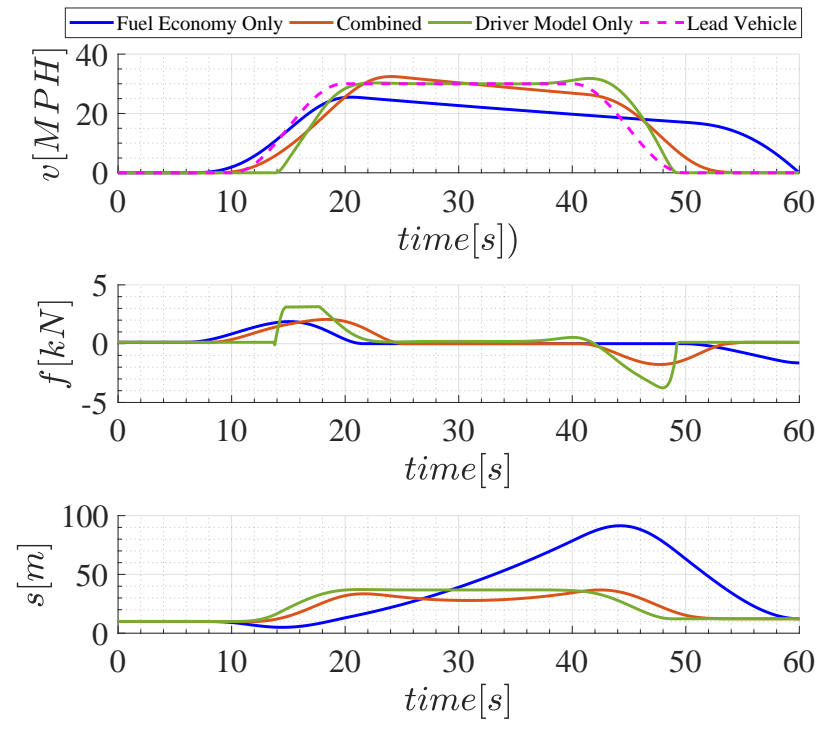

Fig. 5. Speed, traction force and headway distance trajectories for car following case

$w_{2}$ in the cost function $J(19)$ is set as 1 , the optimization problem is solved by varying $w_{1}$ between $10^{-6}$ to $10^{6}$. For each pair of $w_{1}$ and $w_{2}$, we calculated the deviation of the naturalistic driving $\Pi_{\text {nature }}$ and the averaged fuel consumption $\Pi_{\text {fuel }}$. Then the two indexes are plotted in Fig. 4 for all pairs of weighting factors. The trade-off between the driver model cost and the fuel economy are clearly displayed in the Figure. The drivers could save fuel by scarifying their comfort, while the vehicle will consume more fuel by following the drivers' desires.

In Fig. 5, ego-vehicle's speed and force input trajectories for three different weighting factor combination are presented 

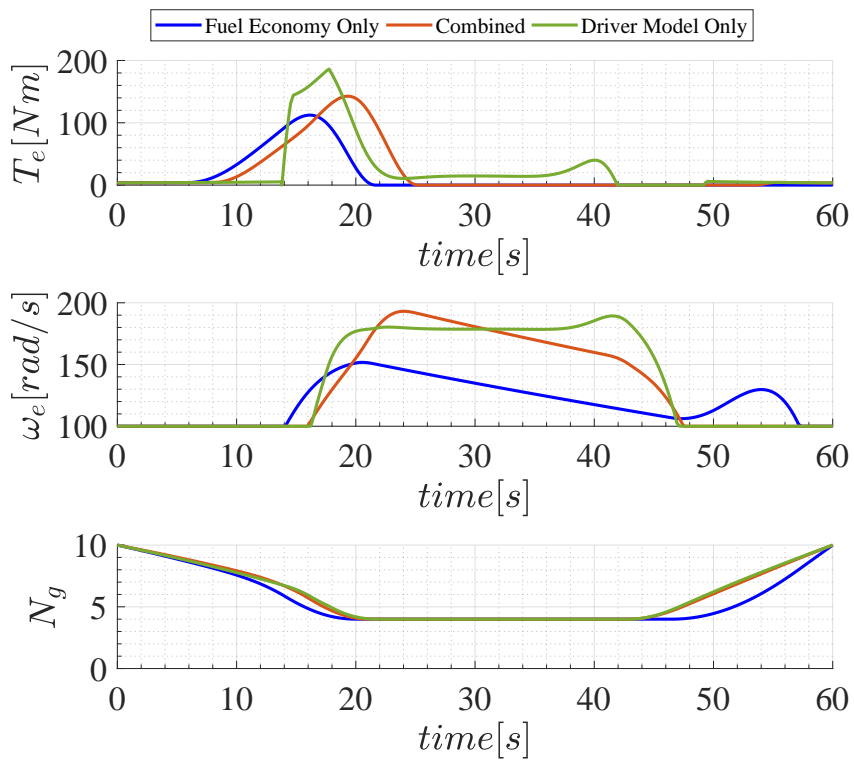

Fig. 6. Engine operation points and CVT ratio for car following case

as well as the headway distance between lead and ego vehicles. The colors of the trajectories in Fig. 5 and Fig. 6 correspond to the filled colored points in Fig. 4. Therefore, the blue trajectories represent the case with the lowest fuel consumption. As shown in the figure, the ego vehicle firstly gently accelerates to a high speed, then the vehicle coasts down between $21 s$ and $51 s$. The headway distance experiences a large variation between $5 \mathrm{~m}$ to $90 \mathrm{~m}$. Even though the fuel economy is lowest of the three cases, the trajectory is not acceptable for a human driver due to the large variation of speed and headway distance.

The green trajectory is the test case that the $\Pi_{\text {nature }}$ is the lowest, which means that the trajectory follows the desires of the driver defined in section II. The speed profile of the egovehicle mimics the behaviour of the lead vehicle, which also results in a headway distance with low variation. However, in this case, the fuel consumption is the highest.

However, the red trajectory strikes a trade-off between the other two test cases. The index $\Pi_{\text {nature }}(0.13[1 / \mathrm{m}])$ is very close to the driver model only test case $(0.12[1 / \mathrm{m}])$, while fuel consumption has reduced from $12.1[\mathrm{~L} / 100 \mathrm{~km}]$ to $10.4[\mathrm{~L} / 100 \mathrm{~km}]$.

In addition, the time series of engine operation points and CVT ratio are shown in Fig. 6. A high CVT ratio is selected during low speed sections to generate large traction forces to the wheel, while a low CVT ratio is selected during the high speed section to make the engine speed stay relatively low for better fuel economy.

\section{B. Case 2: Cornering with No Traffic}

In this part, the curvature of the road is given as a function of the travelled distance as shown in the Fig. 7. So the ego-vehicle travels between two curves with a straight section in the

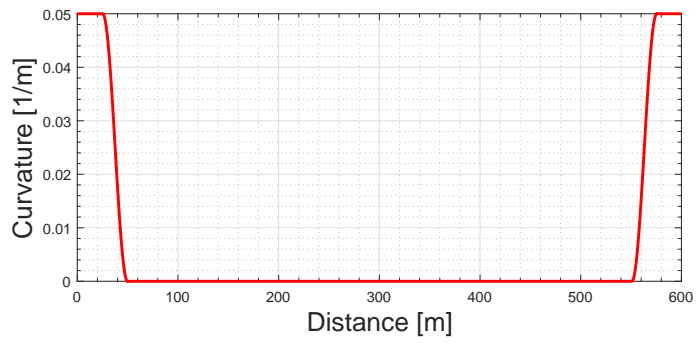

Fig. 7. Curvature vs the travelled distance

middle. The total travel time is $40 \mathrm{~s}$ and the total travel distance is $600 \mathrm{~m}$. Similar to the car following case, the weighting factor $w_{2}$ in the cost function $J(19)$ is set as 1 and the optimization problem is solved by varying $w_{1}$ between $10^{-2}$ to $10^{6}$. For each pair of $w_{1}$ and $w_{2}$, we also calculated the two indexes $\Pi_{\text {nature }}$ and $\Pi_{\text {fuel }}$.

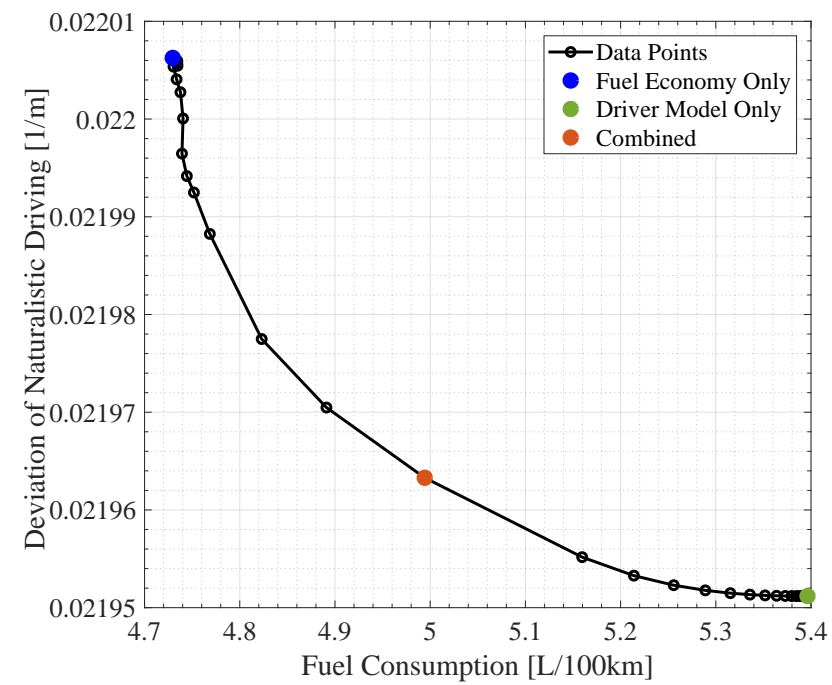

Fig. 8. Trade-off of two weighting factors

The trade-off feature between the naturalistic driving and the fuel economy is presented for the cornering case similarly to the car following case. However, the variation of the index $\Pi_{\text {nature }}$ is much smaller than the car following case. In this subsection, the system state, input and dependent variable trajectories are also plotted for three test cases: fuel economy only $\left(w_{1}=1 e-2\right)$, driver model only $\left(w_{1}=1 e 6\right)$ and combined case $\left(w_{1}=33.9\right)$ and the colors of the trajectories in Fig. 9 and Fig. 10 are corresponding to the filled colored points in Fig. 8.

The trade-off between the naturalistic driving and the fuel economy are clearly displayed in the Figure. The drivers could save fuel by sacrificing their comfort, while the vehicle will consume more fuel by following the drivers' desires. In Fig. 9, ego-vehicle's speed and force input trajectories for three different weighting factor combination are presented as well the headway distance between lead and ego vehicles. The 

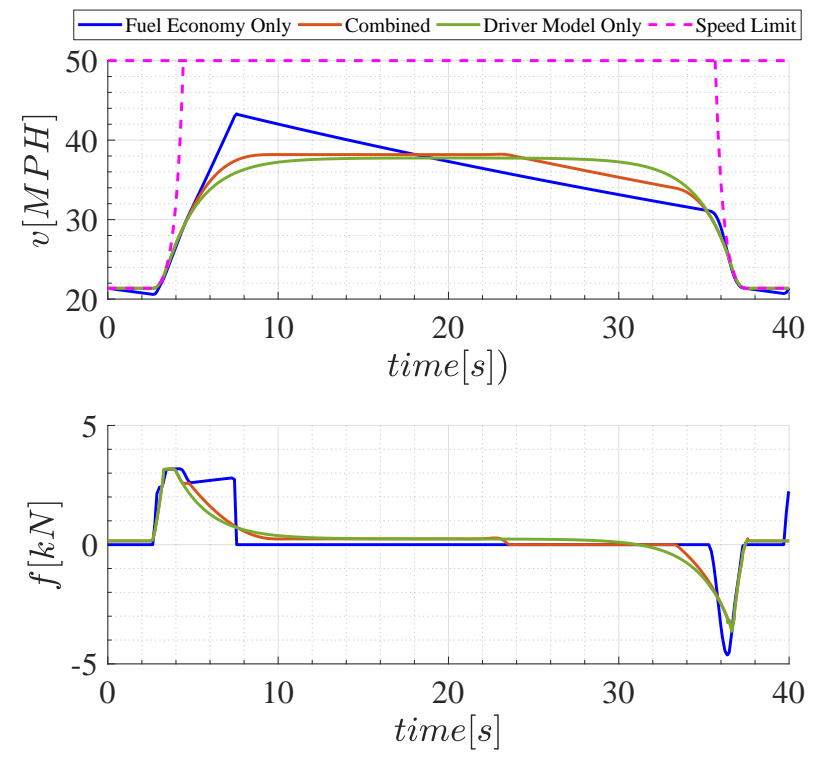

Fig. 9. Speed, traction force and headway distance trajectories for cornering case
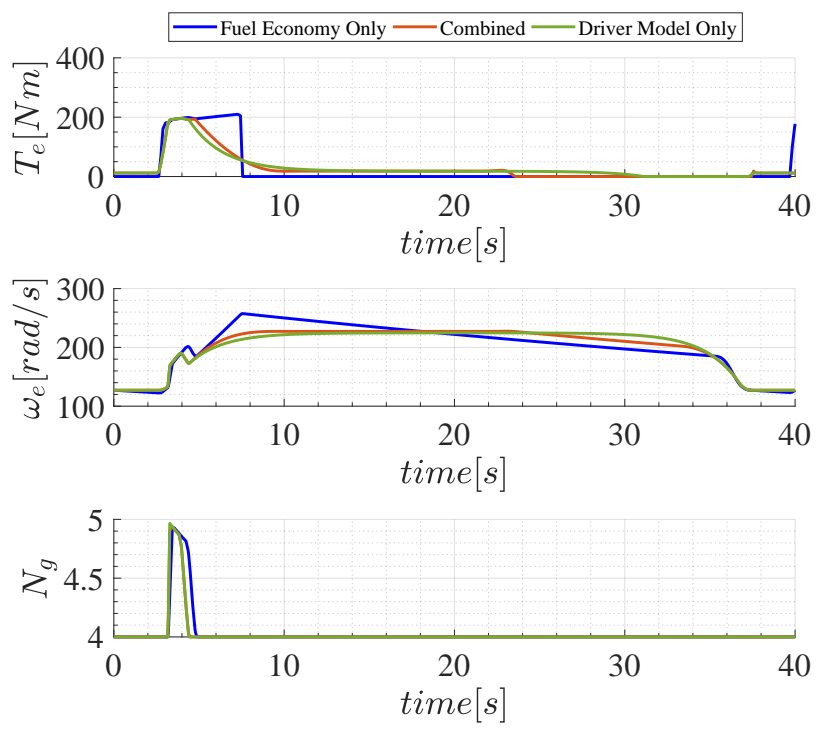

Fig. 10. Engine operation points and CVT ratio for cornering case

colors of the trajectories in Fig. 9 is corresponding to the filled colored points in Fig. 8. For the three test case, the vehicle all travels in low speed due to the curvature constraint (10).

The blue trajectories represent the case with lowest fuel consumption. As shown in the figure, the ego vehicle firstly accelerates to a high speed, then the vehicle coasts down between $7.5 \mathrm{~s}$ and $35.5 \mathrm{~s}$.

The green trajectory is the test case in which the index $\Pi_{\text {nature }}$ is the lowest, which means that the trajectory follows the desires of the driver defined in Section II. Hence, the vehicle accelerates to a constant speed and keeps this speed until the next curve is close, which is the naturalistic way of driving without considering the fuel economy. However, in this case, the fuel consumption is the highest.

The red trajectory is a trade-off between the two test cases above. The value of $\Pi_{\text {index }}$ is very close to the driver model only test case, while the fuel consumption has reduced from $5.4[\mathrm{~L} / 100 \mathrm{~km}]$ to $4.99[\mathrm{~L} / 100 \mathrm{~km}]$. The saving is achieved by coasting down between $23 s$ and 35 .

In addition, the time series of engine operation points and CVT ratio are also shown in Fig. 10. High CVT ratio is selected during the acceleration part in order to generate large traction forces to the wheel.

\section{CONClusion}

A combined optimization problem has been formulated which includes a unified driver model and fuel consumption model for IC engine passenger car. The proposed optimal controller could trade off the driver preference and fuel economy by adjusting the weighting factors. Based on the results, for car following and cornering cases, eco friendly speed and drivetrain input profiles could be given which have low fuel consumption and good user acceptance.

\section{ACKNOWLEDGMENT}

We gratefully acknowledge the support of the Engineering and Physical Research Council under grant number EP/N022262/1.

\section{REFERENCES}

[1] A. af Wåhlberg, "Fuel efficient driving training-state of the art and quantification of effects," 2002.

[2] A. E. af Wåhlberg, "Long-term effects of training in economical driving: Fuel consumption, accidents, driver acceleration behavior and technical feedback," International Journal of Industrial Ergonomics, vol. 37, no. 4, pp. 333-343, 2007.

[3] F. Ding and H. Jin, "On the optimal speed profile for eco-driving on curved roads," IEEE Transactions on Intelligent Transportation Systems, 2018.

[4] A. Sciarretta, G. De Nunzio, and L. L. Ojeda, "Optimal ecodriving control: Energy-efficient driving of road vehicles as an optimal control problem," IEEE Control Systems, vol. 35, no. 5, pp. 71-90, 2015.

[5] A. Schwarzkopf and R. Leipnik, "Control of highway vehicles for minimum fuel consumption over varying terrain," Transportation Research, vol. 11, no. 4, pp. 279-286, 1977.

[6] E. Hellström, J. Åslund, and L. Nielsen, "Design of an efficient algorithm for fuel-optimal look-ahead control," Control Engineering Practice, vol. 18, no. 11, pp. 1318-1327, 2010.

[7] M. A. S. Kamal, M. Mukai, J. Murata, and T. Kawabe, "Ecological vehicle control on roads with up-down slopes," IEEE Transactions on Intelligent Transportation Systems, vol. 12, no. 3, pp. 783-794, 2011.

[8] G. Burnham, J. Seo, and G. Bekey, "Identification of human driver models in car following," IEEE transactions on Automatic Control, vol. 19, no. 6, pp. 911-915, 1974

[9] G. Reymond, A. Kemeny, J. Droulez, and A. Berthoz, "Role of lateral acceleration in curve driving: Driver model and experiments on a real vehicle and a driving simulator," Human factors, vol. 43, no. 3, pp. 483-495, 2001

[10] J. Warga, Optimal control of differential and functional equations. Academic press, 2014

[11] X. Yan, J. Fleming, C. Allison, and R. Lot, "Portable automobile data acquisition module (ADAM) for naturalistic driving study," in Proceedings of the 15th European Automotive Congress, 2017.

[12] M. A. Patterson and A. V. Rao, "GPOPS-II: A MATLAB software for solving multiple-phase optimal control problems using hp-adaptive gaussian quadrature collocation methods and sparse nonlinear programming," ACM Transactions on Mathematical Software (TOMS), vol. 41, no. 1, p. 1, 2014. 